\title{
Factores psicosociales del trabajo (demanda- control y desbalance esfuerzo-recompensa), salud mental y tensión arterial: un estudio con maestros escolares en Bogotá, Colombia*
}

\author{
Psychosocial Job Factors (Demand-Control and Effort-Reward \\ Imbalance), Mental Health and Blood Pressure: A Study \\ with High-School Teachers in Bogotá, Colombia
}

Recibido: marzo 3 de 2009 | Revisado: mayo 19 de 2009 | Aceptado: junio 3 de 2009

\author{
VIVIOLA GÓMEZ-ORTIZ ** \\ LORENA MORENO \\ Universidad de los Andes, Bogotá, Colombia
}

Para citar este artículo. Gómez-Ortiz, V. \& Moreno, L. (2010). Factores psicosociales del trabajo (demanda-control y desbalance esfuerzo-recompensa), salud mental y tensión arterial: un estudio con maestros escolares en Bogotá, Colombia. Universitas Psychologica, 9 (2), 393-407.

Este reporte es el resultado de un estudio empírico realizado en el contexto del grupo de investigación "Estrés y Salud". Parte de los datos se utilizaron para completar la tesis de grado de maestría de la segunda autora. El estudio fue financiado por el departamento de psicología de la Universidad de los Andes y el Centro de Estudios Socioculturales (CESO) de la Facultad de Ciencias Sociales de la misma institución.

** Departamento de Psicología, Carrera 1 No 18A 10, Edificio Franco $2^{\circ}$ piso, Universidad de los Andes, Bogotá, Colombia. Correos electrónicos: vgomez@ uniandes.edu.co; loremz@gmail.com

RES UMEN

Esta investigación identificó la prevalencia de factores psicosociales laborales (FPL), medidos con el JCQ (Job Content Questionnaire) y el ERI (EffortReward Imbalance Questionnaire), en profesores escolares de colegios privados en Bogotá; se evaluó la relación entre estos FPL con salud mental y presión arterial. El estudio fue transversal y correlacional. 251 maestros contestaron los cuestionarios JCQ y ERI y el Cuestionario General de Salud de Goldberg. La presión arterial se midió usando monitores digitales de muñeca. La participación fue voluntaria y anónima. Los resultados mostraron relaciones significativas entre la tensión laboral y el desbalance esfuerzorecompensa con la salud mental pero no con la presión arterial en el grupo completo. Los análisis separados entre hombres y mujeres señalaron algunas relaciones significativas. Los resultados confirman una asociación entre FPL negativos en el contexto laboral de los maestros y su salud mental.

Palabras clave autores

Tensión laboral, desbalance esfuerzo-recompensa, salud mental, presión arterial, profesores escolares.

Palabras clave descriptores

Profesores de educación básica, salud mental, estrés psicológico, presión sanguínea.

\section{A B S T R A C T}

The aims of this research was to identify the prevalence of the psychosocial factors at work, measured with the JCQ (Job Content Questionnaire) and ERI (Effort-Reward Imbalance Questionnaire), in school teachers of Bogotá, and evaluate the relationship of this factors with mental health and blood pressure. This research was crossectional and correlational. A sample of 251 teachers answered the JCQ, the ERI and the General Health Questionnaire. Blood pressure was measured with digital wrist monitors. The participation was voluntary and anonymous. Results showed significant relationships between job strain and effort-reward imbalance with mental health but not with blood pressure for the total group. The results confirm the association between negative psychosocial factor at work with the mental health of school teachers, but not with the blood pressure for all the teachers.

Key words authors

Job Strain, Effort-Reward Imbalance, Mental Health, Blood Pressure, School Teachers. Key words plus

Elementary School Teachers, Mental Health, Stress (Psychology), Blood Pressure. 
Según la Defensoría del Pueblo de Colombia (2008) los estudios sobre salud mental en el país señalan la depresión como uno de los problemas de mayor prevalencia $(19,6 \%)$; otras problemáticas prevalentes son los trastornos de sueño (15\%), la dependencia de sustancias psicoactivas (12\%), el déficit de atención (12\%), el estrés postraumático (6\%) y el pánico (3\%). La ansiedad es una causa o componente importante de varias de ellas. Por otra parte, otro estudio reporta que los trastornos más frecuentemente reportados alguna vez en la vida fueron los de ansiedad (19,3\%), seguido por los trastornos del estado de ánimo $(15,0 \%)$ y los trastornos por uso de sustancias (10,6\%). El 4,9\% de la población estudiada ha intentado suicidarse alguna vez en la vida y el 1,3\% lo ha intentado en los últimos treinta días (Ministerio de la Protección Social de Colombia, 2003; Posada-Villa, AguilarGaxiola, Magaña \& Gómez, 2004).

Por otro lado, la hipertensión arterial primaria o esencial es un problema de salud pública en todo el mundo y constituye un importante factor de riesgo cardiovascular asociado a una mayor probabilidad de eventos cerebro-vasculares y lesiones isquémicas del miocardio. En Colombia, según las últimas estadísticas de la Organización Panamericana de la Salud, la prevalencia de la hipertensión entre la población mayor de 15 años es de 12,6\%. Esta enfermedad constituye el primer factor de riesgo de enfermedades cardiovasculares, las cuales son la segunda causa de muerte en hombres y mujeres mayores de 45 años. Es más, la mortalidad de índole cardiovascular en Colombia alcanza una cifra de 176 por 100.000 habitantes.

Un número importante de investigaciones realizadas en Estados Unidos, Europa y Japón indican que los Factores Psicosociales del Trabajo (FPS-T) afectan la salud y el rendimiento de los trabajadores. La hipertensión arterial es uno de los problemas de salud que se ha señalado como más sensible a los FPS-T (Landsbergis, Schnall, Belkic, Schwartz, Baker \& Pickering, 2008; Schnall, Belkic, Landsbergis \& Baker, 2000). Otros estudios han mostrado como los FPS-T impactan negativamente la salud mental, generando especialmente problemas de ansiedad, depresión, agotamiento, síntomas psicosomáticos y otros (Van der Doef \& Maes, 1999).

Recientemente se aprobó en Colombia la resolución No. 2646 "por la cual se establecen disposiciones y se definen responsabilidades para la identificación, evaluación, prevención, intervención y monitoreo permanente de la exposición a factores de riesgo psicosocial en el trabajo y para la determinación del origen de las patologías causadas por el estrés ocupacional" (Diario oficial, 2008). Algunos de los retos que dicha resolución presenta para los interesados en estas temáticas en Colombia, son validar en el contexto colombiano los modelos e instrumentos ya probados en otros países; elaborar mapas con los factores de riesgo más comunes discriminados por ocupaciones, sectores, géneros, etc.; e investigar las relaciones entre esos factores de riesgo y las consecuencias que acarrean en distintas problemáticas y niveles del funcionamiento humano, tomando en cuenta variables moderadoras y contextuales.

En este contexto, se destaca entonces la necesidad de la investigación del impacto del estrés laboral en algunos indicadores de salud mental y la presión arterial de un grupo de trabajadores colombianos. La salud mental de los maestros, especialmente algunos indicadores, ha sido ampliamente estudiada en algunos países. Por ejemplo, se ha descrito cómo los maestros son particularmente afectados por el síndrome denominado burnout (por ejemplo, Guerrero, 2003; Schaufeli \& Enzmann, 1998; Moreno Jiménez, Garrosa Hernández \& González Gutiérrez, 2000) y otros problemas tales como hábitos de salud (Fernández-Castro \& Doval, 1994) entre otros. Recientemente se realizó en Bogotá un estudio (Diazgranados, González \& Jaramillo, 2006) con maestros del sector público que identificó algunas condiciones laborales y contextuales que afectan negativa y positivamente el bienestar de estas personas. Entre las problemáticas más frecuentemente mencionadas estuvieron las dificultades de concentración, la pérdida de la memoria, beber o fumar en exceso y trastornos del sueño; desde el punto de vista físico se mencionaron dolor de cabeza, fatiga, trastornos vocales, afecciones respiratorias, hipertensión y otros. 
Sin embargo, ninguna de estas problemáticas fue medida por los investigadores; el resultado se basó sólo en lo reportado por los participantes. Hacen falta más estudios que describan de manera más específica los factores psicosociales laborales que se relacionan con la salud de los docentes escolares, especialmente en Colombia.

La salud cardiovascular asociada a los factores psicosociales laborales ha sido una línea de investigación importante en el contexto de la psicología de la salud ocupacional y la medicina del trabajo. Sin embargo, pocas veces se han analizado grupos ocupacionales específicos, y los riesgos particulares de cada grupo que generan un aumento de las dificultades para la salud. Dadas las condiciones de salud en Colombia y el riesgo que en particular parecen correr los maestros escolares, en este estudio se escogió esta ocupación para evaluar el impacto de algunos factores psicosociales laborales en la salud mental percibida y en la tensión arterial.

\section{Los factores psicosociales en el trabajo}

En 1984 el Comité Mixto de la Organización Internacional del Trabajo y la Organización Mundial de la Salud (OIT/OMS) declaró que los factores psicosociales en el trabajo representan el conjunto de las percepciones y experiencias del empleado y abarcan diversos aspectos. El Comité Mixto OIT/ OMS definió:

Los factores psicosociales en el trabajo consisten en interacciones entre el trabajo, su medio ambiente, la satisfacción en el empleo y las condiciones de su organización, por una parte; y por la otra, las capacidades del empleado, sus necesidades, su cultura y su situación personal fuera del trabajo; todo lo cual a través de percepciones y experiencias influyen en la salud y el rendimiento. (p. 3)

Existen modelos avanzados de estrés laboral debido a los FPS-T para comprender y por tanto, predecir los efectos de estos factores sobre la salud. Destacan principalmente, por las evidencias empíricas que los respaldan, el modelo de "Tensión Laboral" o Demanda-Control (D/C) de Karasek (1998) y el modelo de Desequilibrio Esfuerzo-
Recompensa (DER) de Siegrist y Peter (2000). Sin embargo, la investigación con estos modelos ha sido realizada mayoritariamente en países desarrollados (Estados Unidos y algunos países europeos), por lo que se necesitan más estudios representativos con estos modelos en Latinoamérica, pues siguen siendo insuficientes.

En Colombia existen algunos estudios con el modelo demanda-control en poblaciones con diversas ocupaciones (Arango, 2007; Marulanda, 2007) o específicas (Gómez, 2004, 2006; Hernández, Salazar \& Gómez, 2004) que demuestran su validez y la relación de la tensión laboral con indicadores de salud física y mental.

En México, un estudio demostró distintos tipos de validez del modelo de tensión laboral en trabajadoras de una maquiladora en Sonora (Cedillo, 2005); de igual forma, otros estudios latinoamericanos han confirmado la relación del modelo con diversos indicadores de salud (Juárez-García, 2005). La prevalencia de tensión laboral, según estos estudios, es más o menos parecida a la informada en encuestas nacionales de Estados Unidos: 20-25\% (Schnall et al., 2000).

En España se han realizado estudios con el modelo DER encontrando la validez y efectos esperados (Fernández-López, Siegrist, Rödel \& Hernández-Mejía, 2003). En Colombia, Pérez y Gómez (2004) demostraron que el DER discrimina entre sujetos que han experimentado infarto del miocardio y los que no lo han experimentado. Se desconoce el uso y aplicación del DER en otros países latinoamericanos, lo que implica también su urgente estudio y análisis en términos de prevalencia e influencia en la salud en trabajadores de estos países.

Por último, es interesante notar que recientemente se han incrementado los estudios en los que se miden los factores psicosociales laborales, usando simultáneamente los dos modelos descritos previamente puesto que sus autores argumentan que son complementarios y varios estudios así lo han confirmado (Calnan, Wainwrite \& Almond, 2000; Kouvonen, Kivimäki, Cox, Poilainen \& Cox, 2005; Marulanda, 2007; Peter, Siegrist, Hallqvist, Reuterwall \& Theorell, 2002; Siegrist, 2002). 


\section{Factores psicosociales y estrés en los maestros de escuela}

Múltiples son los aspectos que propician el estrés en la labor de los maestros escolares. Entre los estresores que la literatura ha mencionado se encuentran las dificultades con los recursos, la disciplina en el aula, los conflictos y la ambigüedad en el rol, la sobrecarga de trabajo, el bajo reconocimiento profesional y la interrelación con los otros maestros. Por ejemplo, Oramas, Rodríguez, Almirall, Huerta y Vergara (2003, p. 8) reportan que "Las demandas de trabajo-multiplicidad de tareas, responsabilidades con el alumnado, la relación con los padres, etc. -y el comportamiento de los estudiantes-irrespetuoso, bullicioso, difícilse destacan en una relación directa con el estrés en maestros". Un clima organizacional en el que predomine el proceso de crossover-irradiación- de la tensión del equipo de dirección hacia los subordinados y viceversa, puede generar una reacción circular de tensión, que trae como consecuencia condiciones estresantes para los maestros. En un estudio reciente, otros autores reportan como estresores de la labor del maestro, la carga de trabajo, considerando ésta una función directa de las condiciones del ambiente físico de trabajo -ruido, iluminación- y el mobbing o psicoterror laboral (Boyle \& Borg, 1995; Cooper \& Kelly, 1993; Kyriacou, 1989; Westman \& Etzion, 1999; citados en Oramas et al., 2003).

Desde otra perspectiva teórica, utilizando el Occupational Stress Index [OSI], se han descrito y cuantificado algunas de las condiciones laborales de los maestros. Belkic (2000) ha descrito algunos de los aspectos demandantes de las tareas de los maestros tales como deber prestar atención a múltiples fuentes de información simultáneamente, la necesidad de tomar decisiones complejas y complicadas, procesar nueva información rápidamente, trabajar bajo presión de tiempo, conflicto e incertidumbre relacionada con el tamaño de los cursos y las demandas curriculares así como con las tareas administrativas, interrupciones relacionadas con cambios de planes en el trabajo, etc.
En Colombia, Diazgranados et al. (2006) realizaron un estudio con 562 docentes de 25 instituciones educativas distritales (sector público) de Bogotá. En este estudio realizado "con el ánimo de determinar cuáles son las principales problemáticas que rodean el quehacer de los docentes del Distrito, el impacto que tienen sobre su bienestar psicosocial, y los saberes y habilidades que usan para minimizar sus efectos negativos" (p. 45), se encontró que el horario laboral, las horas de clase asignadas y el volumen de tareas, entre otras, fueron señaladas por la gran mayoría de los participantes como condiciones positivas. Otros factores como el tiempo de desplazamiento que usan entre la residencia y la institución, el tiempo con que cuentan para preparar el trabajo y el clima laboral en la institución también fueron evaluados positivamente. Sin embargo, aspectos del contexto laboral tales como el salario devengado, el número de alumnos por aula, las oportunidades de ascenso laboral y los criterios de evaluación se consideraron por la mayoría como aspectos que no favorecen su bienestar. En cada institución, entre más percibieron los docentes que había aspectos del contexto laboral que no beneficiaban su bienestar, hubo mayor reporte de malestar psicológico y físico. Se encontró que las características de la población estudiantil atendida tienen el impacto más negativo sobre el bienestar docente. Asuntos tales como la disciplina, el comportamiento de los alumnos dentro y fuera del aula, la motivación y compromiso de los alumnos y de sus familias y la situación socioeconómica de los alumnos afectan negativamente el bienestar y la salud de los docentes.

Las autoras (Diazgranados et al., 2006) concluyeron en su trabajo que a pesar de que, efectivamente, hay algunas condiciones del contexto laboral que afectan la salud física y mental de los maestros, este efecto negativo parece ser menor del que se dice y circula como un "relato dominante" en la ciudad. Efectivamente, la mayor parte de los participantes perciben que en general los demás están muy mal, especialmente desde el punto de vista mental, mientras que personalmente no reportan un malestar tan serio. Pareciera haber una 
discrepancia "entre la descripción negativa que los docentes brindan sobre sus compañeros y el panorama alentador que obtuvimos en los cuestionarios" (p. 53). Las autoras interpretan esta discrepancia como un uso político, una forma de lograr un impacto en la opinión pública y el Gobierno, "contribuyendo así a fortalecer el imaginario de que se están enfermando mentalmente por las difíciles condiciones laborales en que ejercen su profesión" (p. 54). A pesar de lo anterior, no se desconoce que hay condiciones laborales (especialmente en lo referente a lo exigente, demandante y con poco reconocimiento que tiene la labor de maestro escolar en el país) que ameritan recomendaciones como "promover iniciativas de reconocimiento social a los docentes y apoyar la implementación de espacios generativos de participación y trabajo en equipo como parte de la labor remunerada de los docentes" (p. 54), entre otras.

\section{Objetivos de la presente investigación}

Los objetivos del presente estudio fueron: 1) identificar la prevalencia de los factores psicosociales citados (Tensión laboral y Desbalance esfuerzorecompensa) en una muestra de maestros escolares de Bogotá, Colombia; y, 2) determinar la asociación de estos factores psicosociales con indicadores de salud mental y tensión arterial.

\section{Método}

\section{Diseño}

Esta fue una investigación no experimental, correlacional y transversal. Se obtuvo información acerca de aspectos sociodemográficos de los y las participantes, tales como: institución de trabajo, ocupación, antigüedad en el puesto, estado civil, sexo y edad. Se exploraron factores tradicionales de riesgo para la tensión arterial elevada (peso y estatura, sedentarismo, tabaquismo, alcoholismo, dieta y enfermedades tales como diabetes). Aquellos que correlacionaron con la tensión arterial se controlaron estadísticamente.

\section{Participantes}

Se seleccionaron de manera no probabilística y por conveniencia, 251 maestros de distintos colegios privados de estrato alto y medio-alto de la ciudad de Bogotá (edad promedio de 35.4 años, DS 8.3, rango de 21 a 63 años).

El 23.1\% de la muestra (58 personas) eran del sexo masculino y el 76.9\% (193 personas) del sexo femenino. Los maestros participantes desempeñaban labores de docencia en todos los grados del nivel primario y medio de educación ( 5 a 16 años). La mayor parte de los maestros a los que se les invitó a participar en el estudio aceptó voluntariamente la invitación (95\%). Esta participación fue anónima.

El tiempo promedio que llevaban trabajando en la profesión fue de 8.9 años (DS 6.9). El 63.3\% de los maestros trabaja 40 horas a la semana; $15.9 \%$ trabaja entre 10 y 38 horas; finalmente, el $20.7 \%$ trabaja entre 42 y 65 horas a la semana. El 42.2\% desempeña otras labores remuneradas además de su labor docente. El 94.9\% de la muestra tenía educación universitaria (61.8\%) o posgraduada (33.1\%). Los demás tenían formación tecnológica (4.4\%) o académica media (bachillerato).

\section{Instrumentos}

Se construyó una encuesta con el siguiente contenido:

1ํ. El Cuestionario del Contenido del Trabajo desarrollado por Karasek (1985) (JCQ, por sus siglas en inglés) y traducido al castellano por Cedillo (1999). Este cuestionario evalúa los factores psicosociales del trabajo postulados en el modelo demanda-control-apoyo social. El JCQ-Center, administrador oficial del JCQ, otorgó autorización a la investigadora principal para el uso de este instrumento. De esta traducción, que contiene 42 ítems, se utilizaron los 27 que corresponden a la versión en inglés con este número de preguntas. Las confiabilidades de las escalas del JCQ (calculadas con el alfa de Cronbach) con este grupo, fueron las siguientes: uso de habilidades (6 ítems, rango 12-48, $\alpha$ 0.6); toma de decisiones ( 3 ítems, rango 12-48, 
$\alpha$ 0.6); control de procesos laborales (9 ítems, rango 24-96, $\alpha$ 0.7); demandas psicológicas (5 ítems, rango 12-48, $\alpha$ 0.7); apoyo de Jefe (4 ítems, rango 4-16, $\alpha$ 0.8); apoyo de colegas (4 ítems, rango 4-16, $\alpha$ 0.8); inseguridad laboral (3 ítems, rango 3-12, $\alpha$ 0.6). La tensión laboral puede calcularse de varias maneras. Las que son posibles de calcular en este caso son dos: un valor continuo equivalente a la relación entre demandas y latitud de decisión; la segunda opción es calcular, con base en las medianas de los valores de demanda y latitud de decisión, las cuatro combinaciones posibles con los valores de demanda y latitud de decisión. El grupo que representa la mayor tensión laboral es el que combina altos valores de demandas y bajos valores de latitud de decisión.

2‥ El Cuestionario de Desequilibrio EsfuerzoRecompensa (DER) desarrollado por Siegrist (2000) y traducido al castellano por Fernández et al. (2003). Este fue adaptado para la población colombiana por Pérez y Gómez (2004). Las confiabilidades de las escalas del ERI (calculadas con el alfa de Cronbach) con este grupo fueron las siguientes: esfuerzo extrínseco (5 ó 6 ítems, en este estudio se usaron 5 por lo que el rango osciló entre 5 y 25, $\alpha$ 0.8); recompensas (11 ítems, rango 11-55, $\alpha$ 0.8); sobrecompromiso (6 ítems, rango $6-24, \alpha 0.8$ ). Mayores puntajes en cada subescala indican mayores esfuerzos extrínsecos, recompensas y sobrecompromiso. 3‥ El Cuestionario General de Salud (GHQ, por sus siglas en inglés), en su versión de 28 reactivos. Este instrumento es utilizado para evaluar la percepción de salud mental y física. Las subescalas de este instrumento son: disfunción social, ansiedad, depresión y síntomas psicosomáticos. Cada escala está compuesta por 7 ítems, el rango posible de valores en cada subescala oscila entre 7 y 28, y los alfas de Cronbach obtenidos en este estudio fueron 0.7, 0.9, 0.9 y 0.9 , respectivamente. Un mayor puntaje en cada subescala o en la escala total es un indicador de peores condiciones de salud.

4ํ․ Un formato de datos generales, sociodemográficos y ciertos aspectos de salud.
Para la medición de Tensión Arterial se usaron monitores digitales de muñeca marca OMRON Modelo HEM-609INT. Estos monitores tienen una garantía de calibración indefinida y han sido valorados por diversas asociaciones (European Society of Hypertensión y Association for the Advancement of Medical Instrumentation) como suficientemente confiables de acuerdo con protocolos internacionales. Además, utilizados para fines de investigación han mostrado resultados adecuados y aceptables (Schnall \& Belkic, 2000).

\section{Procedimiento}

Una vez la dirección de cada colegio contactado daba su autorización para realizar el estudio, se invitó a los maestros a una reunión en la que se les explicó el proyecto y su forma de participación. A quienes aceptaron se les pidió su consentimiento firmado y se les entregó el cuestionario que debían completar antes de la siguiente cita. Varios días después, la tensión arterial (TA) fue medida durante un día laboral normal. Esta medición se realizó de acuerdo con el "protocolo de puntos estimados en el trabajo" recomendado por Schnall (véase, Schnall \& Belkic, 2000) cuyas ventajas son que se captura la TA más representativa y real que tiene una persona durante el día (en el trabajo) y el promedio no se diferencia significativamente del que se obtiene en la TA ambulatoria (Schnall \& Belkic). De cada maestro se obtuvieron dos series de mediciones: una (dos medidas separadas por un minuto) al principio de la primera pausa de la mañana; por la tarde, después del almuerzo, se realizó la segunda serie de mediciones. En todos los casos, mientras se hicieron las mediciones, los maestros estuvieron de pie como suelen estarlo durante sus labores docentes. Todas las evaluaciones se hicieron en el mes de junio de 2008, cuando todos los colegios se encontraban terminando labores antes de las vacaciones de mitad del año escolar.

Para el análisis de los datos se utilizaron estadísticas descriptivas tales como medias, desviaciones estándar, frecuencias y porcentajes, para estimar prevalencias y observar comportamiento general de los datos. Para analizar la relación entre varia- 
bles se utilizaron estadísticas multivariadas tales como la correlación y la regresión múltiple mediante el programa SPSS (versión 16).

\section{Resultados}

\section{Análisis descriptivos de la muestra}

Los valores medios en las escalas del cuestionario general de salud (GHQ-28) fueron los siguientes: Psicosomatización ( $M=17.4$, DS 5.1); Ansiedad $(M=15.4$, DS 5.8); Disfunción social $(M=15.7$, DS 3.3); Depresión ( $M=8.6$, DS 3); Puntaje total $(\mathrm{M}=57.1, \mathrm{DS} 12.9)$. Estos valores indican que el problema de salud más frecuente fue la psicosomatización, seguido de la disfunción social y la ansiedad. La frecuencia de indicadores de depresión en esta muestra es baja.

Los promedios de tensión arterial sistólica y diastólica obtenidos estuvieron dentro de los rangos normales $(M=115.5$, DS $13 / M=73.7$, DS 9 $\mathrm{mm} \mathrm{Hg}$ ). Los factores biomédicos de riesgo para la hipertensión arterial se presentaron en las siguientes proporciones: diabetes (4.4\%), tabaquismo (15.9\%), sedentarismo (31.9\%), consumo de alcohol mensual o semana (5.2\%). El 48.2 \% de los participantes reportó no tener antecedentes familiares de hipertensión arterial, el 40\% reportó antecedentes en uno de sus padres o en los dos, y el resto indicó que uno de sus abuelos o un hermano(a) padecía de hipertensión. El valor promedio del Índice de masa corporal (IMC) indica que esta población no presenta problemas importantes de sobrepeso (22.7, DS 3.2).

De los factores de riesgo biomédico sólo el IMC se asoció positivamente con los niveles de tensión arterial. Entre las variables demográficas, la edad correlacionó positivamente con los niveles de tensión arterial. La tensión sistólica y diastólica de los hombres fue significativamente más alta que la de las mujeres (125.3 vs. $112.8 / 79.8$ vs. $71.8, p<.01$ ).

Por otro lado, 11 Personas (4.5\%) reportaron padecer de hipertensión. Según nuestras medidas, 8 personas (3.2\%) presentaron hipertensión (valores iguales o superiores a $140 / 90 \mathrm{~mm} \mathrm{Hg}$ ) y $18(7.2 \%)$ hipertensión sistólica solamente (va- lores superiores a $140 \mathrm{~mm} \mathrm{Hg}$ ). Las personas que manifestaron padecer hipertensión presentaron efectivamente una tensión arterial sistólica significativamente más alta que el resto de los participantes $(p<.01)$.

\section{Prevalencia de los factores psicosociales laborales}

Los análisis descriptivos (véase Tabla 1) mostraron que los promedios de las escalas "uso de habilidades", "toma de decisiones", "control de los procesos laborales", "demandas laborales" e "inseguridad laboral" son más altos que los obtenidos en muestra nacionales e internacionales; los promedios de "apoyo de jefes y colegas" están dentro del promedio.

Utilizando como indicador de tensión laboral la razón entre las demandas y la latitud de decisión (control), calculada según la fórmula propuesta por el JCQ-Center $<($ Demands *2)/Decision-Latitude) $>$, el $45.8 \%$ de los maestros tienen valores de tensión laboral por debajo del puntaje de riesgo $(<1)$ y el $54.2 \%$ están por encima de dicho valor $(>1)$. Si se utiliza como criterio de clasificación la combinación de altas o bajas demandas y alto o bajo control, el resultado del número de docentes que clasifica en cada uno de los cuadrantes del modelo Demanda-Control es el siguiente: Trabajo de alta tensión: 87 (35.5\%); Trabajo activo: 36 (14.7\%); Trabajo de baja tensión: 69 (28.2\%); Trabajo pasivo: 53 (21.6\%).

Por otro lado, los valores de esfuerzo y de sobrecompromiso, escalas del cuestionario DER, fueron sensiblemente más altos que los obtenidos con otras muestras colombianas y españolas. El valor promedio en este último componente (sobrecompromiso) es similar al reportado por las enfermeras. De la misma manera, las recompensas tienden a ser altas, similares a las de las enfermeras y uno de los grupos ocupacionales mixtos (véase Tabla 2). Según Siegrist (2004), un valor superior a 1 al calcular la razón entre esfuerzo y recompensa, indica un desbalance entre éstos a favor de los esfuerzos lo que se constituiría en un factor de riesgo para la salud. Según este indicador, el $77.7 \%$ de la muestra 
TABLA 1

Medias de los maestros en las escalas del JCQ comparadas con las de otros grupos colombianos y no colombianos

\begin{tabular}{|c|c|c|c|c|c|c|c|c|c|c|c|c|}
\hline \multirow[t]{2}{*}{ Escala } & \multicolumn{2}{|c|}{$\begin{array}{l}\text { Maestros } \\
\text { colomb. }\end{array}$} & \multicolumn{2}{|c|}{$\begin{array}{c}\text { Enfermeras } \\
\text { colomb. }+\end{array}$} & \multicolumn{2}{|c|}{$\begin{array}{l}\text { Choferes } \\
\text { colomb.* }\end{array}$} & \multicolumn{2}{|c|}{$\begin{array}{l}\text { Ocup. Mix. } \\
\text { col. \& }\end{array}$} & \multicolumn{2}{|c|}{ Internal. \# } & \multicolumn{2}{|c|}{ México \% } \\
\hline & M & DS & M & DS & M & DS & M & DS & $\mathbf{M}$ & DS & M & DS \\
\hline Uso de habilidades & 38.7 & 4.3 & 37.3 & 4.9 & 32 & 6.2 & 36.7 & 5.22 & 32.7 & 7.7 & 34.5 & 4.8 \\
\hline Autoridad de decisión & 33.8 & 6.8 & 33.3 & 6.4 & 28 & 7.2 & 32.85 & 5.53 & 32.5 & 8.4 & 32.4 & 7.3 \\
\hline Control & 72.5 & 9.6 & 70.6 & 9.8 & 61.9 & 9.8 & 69.45 & 8.96 & & & & \\
\hline Demandas psicológicas & 36.3 & 6.8 & 34 & 3.7 & 32.6 & 6.4 & 33.7 & 4.35 & 32 & 7.3 & 29.3 & 6.4 \\
\hline Demandas físicas & & & 2.9 & 0.8 & 2.8 & 0.9 & 2.03 & 0.9 & 2.3 & 1.1 & 2.3 & 0.85 \\
\hline Apoyo social colegas & 12.3 & 2.1 & 12 & 2 & 11.3 & 2.1 & 12.3 & 2.25 & 12.4 & 2.6 & 12.2 & 2.1 \\
\hline Apoyo social jefe & 11.7 & 2.5 & 10.9 & 2.4 & 9.7 & 2.8 & 11.67 & 2.56 & 11.9 & 3.2 & 11.2 & 2.4 \\
\hline Inseguridad laboral & 6.3 & 2 & 7 & 2.3 & 6.3 & 2.4 & 6 & 1.98 & 3.7 & 1.5 & 3.3 & 0.96 \\
\hline
\end{tabular}

+y * Arango, 2007; \& Marulanda, 2007; \# Karasek et al. (1998); \% Cedillo et al. (2003).

Fuente: elaboración propia.

no percibe desbalance entre el esfuerzo y la recompensa, y el $22.3 \%$ sí lo percibe.

\section{Relación entre los factores psicosociales laborales e indicadores de salud}

Para empezar es importante señalar que la correlación entre los dos indicadores de estrés laboral, la tensión laboral y el desbalance esfuerzo-recompensa fue alta $(r=0.61)$ y significativa $(p<.001)$, lo cual señala que ambos indicadores están midiendo un constructo similar, pero no idéntico.
Las correlaciones entre los factores psicosociales (valor continuo de tensión laboral y de desbalance esfuerzos-recompensas) y los indicadores de salud mental fueron positivas y significativas $(p<0.001)$ en todos los casos, exceptuando la correlación entre disfunción social y el DER. Esto indica que con la excepción señalada, entre más percepción de estrés laboral hay, esta muestra tiende a percibir también mayor depresión, ansiedad, disfunción social y síntomas psicosomáticos (véanse Tablas 3 y 4). Vale la pena destacar el alto valor de las correlaciones entre demandas y ten-

TABLA 2

Medias de los maestros en las escalas del DER comparadas con las de otros grupos colombianos y una muestra española

\begin{tabular}{|c|c|c|c|c|c|c|c|c|c|c|c|c|}
\hline \multirow[t]{2}{*}{ Escala } & \multicolumn{2}{|c|}{$\begin{array}{l}\text { Maestros } \\
\text { colomb. }\end{array}$} & \multicolumn{2}{|c|}{$\begin{array}{l}\text { Enfermeras } \\
\text { colomb. }+\end{array}$} & \multicolumn{2}{|c|}{$\begin{array}{l}\text { Choferes } \\
\text { colomb.* }\end{array}$} & \multicolumn{2}{|c|}{$\begin{array}{l}\text { Ocup. Mixtas } \\
\text { colomb. } 1 \&\end{array}$} & \multicolumn{2}{|c|}{$\begin{array}{c}\text { Ocup. Mixtas } \\
2 \#\end{array}$} & \multicolumn{2}{|c|}{ España \% } \\
\hline & $\mathbf{M}$ & DS & $\mathbf{M}$ & DS & $\mathbf{M}$ & DS & $\mathbf{M}$ & DS & $\mathbf{M}$ & DS & $\mathbf{M}$ & DS \\
\hline & $M$ & DS & $\mathrm{M}$ & DS & $M$ & DS & $\mathrm{M}$ & DS & $M$ & DS & $\mathrm{M}$ & DS \\
\hline Esfuerzo & 17.6 & 5.5 & 14.9 & 5 & 15.1 & 6.1 & 14.3 & 4.5 & 13.1 & 0.4 & 15.6 & 4 \\
\hline Recompensa & 43.7 & 8.7 & 43.7 & 9.5 & 28.9 & 3.8 & 39.82 & 10.9 & 46.6 & 8.5 & 35.2 & 9.4 \\
\hline Sobrecompromiso & 18 & 4.1 & 14.3 & 3.3 & 15.3 & 3.9 & 14.5 & 2.9 & 14.7 & 3.3 & 2.42 & 0.5 \\
\hline
\end{tabular}

+ y* Arango, 2007; \& Marulanda, 2007; \# Pérez y Gómez (2005); \% Macías et al. (2003).

Fuente: elaboración propia. 
sión laboral por un lado, y los componentes del modelo DER y su indicador de balance por otro con ansiedad, psicosomatización y el indicador general de salud.

Indicadores de condiciones laborales tales como tiempo desempeñando el actual trabajo, tener turnos o no, desempeñar otros trabajos además del principal y horas remuneradas a la semana, no mostraron una relación significativa con ninguna de las medidas de salud evaluadas.
Las medidas globales de estrés laboral (Tensión laboral o DER) ni sus componentes individuales correlacionaron con la tensión arterial (sistólica o diastólica). En estas correlaciones se controlaron la edad, el sexo y el índice de masa corporal, puesto que todos ellos correlacionaron significativamente con la tensión arterial.

Otras formas de analizar la relación entre la presión arterial y la tensión laboral propuesta por el modelo Demanda-Control, fue comparar los

TABLA 3

Correlaciones entre variables del modelo de estrés laboral Demanda -control-apoyo social y salud

\begin{tabular}{|c|c|c|c|c|c|c|c|c|c|}
\hline & $\begin{array}{l}\text { Habili- } \\
\text { dades }\end{array}$ & $\begin{array}{l}\text { Decisio- } \\
\text { nes }\end{array}$ & Control & $\begin{array}{l}\text { Deman- } \\
\text { das }\end{array}$ & $\begin{array}{l}\text { Apoyo } \\
\text { jefe }\end{array}$ & $\begin{array}{c}\text { Apoyo } \\
\text { Compa- } \\
\text { ñeros }\end{array}$ & $\begin{array}{l}\text { Apoyo } \\
\text { Social }\end{array}$ & $\begin{array}{l}\text { Inseguri- } \\
\text { dad }\end{array}$ & Job strain \\
\hline Salud & $-.173(* *)$ & $-.346(* *)$ & $-.324(* *)$ & $.449(* *)$ & $-.242(* *)$ & $-.265(* *)$ & $-.298(* *)$ & $.220(* *)$ & $.498(* *)$ \\
\hline $\begin{array}{l}\text { Psicosomá- } \\
\text { ticos }\end{array}$ & -.124 & $-.301(* *)$ & $-.270(* *)$ & $.435(* *)$ & $-.251(* *)$ & $-.218(* *)$ & $-.279(* *)$ & $.183(*)$ & $.451(* *)$ \\
\hline Ansiedad & -.061 & $-.278(* *)$ & $-.226(* *)$ & $.465(* *)$ & $-.161(*)$ & $-.225(* *)$ & $-.225(* *)$ & $.217(* *)$ & $.466(* *)$ \\
\hline $\begin{array}{l}\text { Disfunción } \\
\text { social }\end{array}$ & $-.198(* *)$ & $-.178(* *)$ & $-.215(* *)$ & $.161\left(^{*}\right)$ & -.102 & $-.167(* *)$ & $-.155(*)$ & .070 & $.226(* *)$ \\
\hline Depresión & $-.195(* *)$ & $-.233(* *)$ & $-.253(* *)$ & .106 &. $.184(* *)$ & $-.146(*)$ & $-.197(* *)$ & $.133(*)$ & $.214(* *)$ \\
\hline Tensión Sistólica & -.162 & -.022 & -.088 & -.112 & -.040 & -.063 & -.060 & .003 & -.026 \\
\hline $\begin{array}{l}\text { Tensión } \\
\text { Diastólica }\end{array}$ & -.101 & .000 & -.045 & -.058 & -.026 & .018 & -.027 & -.035 & -.003 \\
\hline
\end{tabular}

Variables controladas en las correlaciones con tensión sistólica y diastólica: Edad, Sexo e IMC.

Fuente: elaboración propia.

TABLA 4

Correlaciones entre variables del modelo de estrés laboral Esfuerzo-Recompensa y salud

\begin{tabular}{lcccc}
\hline & Esfuerzo & Recompensa & Sobrecompromiso & DER \\
\hline Salud & $.560(* *)$ & $-.422(* *)$ & $.649(* *)$ & $.559(* *)$ \\
Psicosomáticos & $.516(* *)$ & $-.394(* *)$ & $.588(* *)$ & $.511(* *)$ \\
Ansiedad & $.603(* *)$ & $-.435(* *)$ & $.679(* *)$ & $.599(* *)$ \\
Disfunciónsocial & .120 & -.094 & $.232(* *)$ & .122 \\
Depresión & $.219(* *)$ & $-.188(* *)$ & $.206(* *)$ & $.227(* *)$ \\
Tensión Sistólica & .065 & -.067 & -.019 & .056 \\
Tensión Diastólica & .046 & -.049 & -.001 & .034 \\
\hline
\end{tabular}

Variables controladas en las correlaciones con tensión sistólica y diastólica: Edad, Sexo e IMC.

Fuente: elaboración propia. 
niveles de tensión arterial de las personas pertenecientes a los cuatro cuadrantes del modelo; estos grupos no se diferenciaron significativamente en sus niveles de tensión arterial. Grupos con niveles extremos de tensión laboral, calculados con base en cuartiles y tertiles, tampoco se diferenciaron en sus niveles de tensión arterial. Por último, se conformaron 8 grupos utilizando para ello las medianas de control, demanda y apoyo social (modelo Demanda-Control-Apoyo), pero esta forma de clasificación tampoco arrojó ninguna relación entre el nivel de estrés laboral y la tensión arterial. Vale la pena anotar que ninguno de los indicadores de salud medidos (psicosomatización, ansiedad, disfunción social o depresión) correlacionó con la tensión arterial.

Con el fin de evaluar qué tanto aporta cada uno de los modelos de estrés laboral a la explicación de la varianza de la salud mental, se realizó un análisis de regresión. El resultado señala que entre ambos indicadores explican aproximadamente el 35\% de la varianza de la salud mental. Sin embargo, el modelo DER tiene un impacto mayor que el de Demanda-Control, lo cual se puede apreciar en la Tabla 5 en los valores de las Betas.

\section{TABLA 5}

Regresiones de los modelos de estrés laboral sobre el indicador general de salud

\begin{tabular}{lccc}
\hline \multicolumn{1}{c}{ Variable } & B & SE B & $\beta$ \\
\hline Tensión laboral & 12.2 & 2.0 & $.252^{* *}$ \\
DER & 13.0 & 3.1 & $.406^{* *}$ \\
$\mathrm{R}^{2}$ & & $.352^{* *}$ & \\
$\mathrm{~F}$ & & 67.4 & \\
\hline
\end{tabular}

$* * p<.01$.

Fuente: elaboración propia.

Finalmente, se consideró pertinente evaluar la relación entre el estrés laboral y sus indicadores globales con la tensión arterial de manera separada en cada sexo (controlando la edad y el IMC), debido a la diferencia significativa entre hombres y mujeres en el nivel de tensión arterial y a que la diferencia en el tamaño de los grupos por sexo fue grande. Este análisis arrojó como resultado, en los hombres, una correlación negativa y significativa entre el nivel de recompensas percibido con el nivel de tensión arterial sistólica $(r=-.351, p<.01)$, y una correlación positiva entre el desbalance esfuerzo-recompensa y la tensión arterial sistólica $(r=.270, p<.05)$. En otras palabras, entre menos recompensas y más desbalance esfuerzo-recompensa percibieron los docentes hombres, mayor fue el nivel de tensión arterial sistólica que presentaron. En el caso de las mujeres, el mismo análisis mostró una correlación negativa y significativa entre el subcomponente del control laboral, uso de habilidades, y la tensión sistólica $(r=-.162, p<.05)$. Es decir, que entre más usan las mujeres sus habilidades en el trabajo, menor es la tensión sistólica que presentan.

Con la tensión arterial sistólica se realizaron dos regresiones, una para cada sexo, en la que se evaluaron simultáneamente todas las variables que en cada caso mostraron una relación con ella. En el caso de los hombres, resultó que las únicas variables que explican parte de su varianza (12\%) fueron las recompensas y el desbalance esfuerzorecompensa, siendo la recompensa el factor más determinante (véase, Tabla 6).

\section{TABLA 6}

Regresiones de algunos indicadores de estrés laboral sobre la tensión arterial en hombres

\begin{tabular}{lccc}
\hline \multicolumn{1}{c}{ Variable } & B & SE B & B \\
\hline Modelo 1 & & & \\
Edad & -0.58 & .190 & -.041 \\
IMC & .853 & .619 & .186 \\
$R^{2}$ & & & .034 \\
$F$ & & & .963 \\
Modelo 2 & & & \\
Edad & -0.40 & .187 & .028 \\
IMC & .671 & .603 & .146 \\
Recompensas & -.454 & .299 & .319 \\
DER & .593 & 6.88 & .318 \\
Cambio en $R^{2}$ & & & $0.11 *$ \\
F & & & 2.18 \\
\hline$* * p<.01 ; * p<.05$. & & &
\end{tabular}

Fuente: elaboración propia. 
En el caso de las mujeres, la regresión mostró que tanto la edad como el IMC y el uso de habilidades, contribuyen a explicar un $18 \%$ de la varianza en la tensión arterial sistólica. La edad y el IMC explican el 15\% de dicha variable y el factor psicosocial laboral, un 3\% adicional (véase Tabla 7). En el caso de las mujeres, la edad fue el factor más determinante.

\section{TABLA 7}

Regresiones de algunos indicadores de estrés laboral sobre la tensión arterial en mujeres

\begin{tabular}{lccc}
\hline \multicolumn{1}{c}{ Variable } & B & SE B & B \\
\hline Modelo 1 & & & \\
Edad & .464 & .102 & $.319^{* *}$ \\
IMC & .572 & .249 & $.160^{*}$ \\
$R^{2}$ & & & .15 \\
F & & & $16.2^{* *}$ \\
Modelo 2 & & & \\
Edad & .490 & .101 & $.337^{* *}$ \\
IMC & .618 & .247 & $.173^{*}$ \\
Uso de &. .447 & .182 &. $.167^{*}$ \\
habilidades & & & .03 \\
Cambio en $R^{2}$ & & & $13.1^{* *}$ \\
F & & & \\
\hline
\end{tabular}

$* * p<.01 ; * p<0.5$.

Fuente: elaboración propia.

\section{Discusión}

Los hallazgos de este estudio confirmaron que los maestros escolares colombianos presentan un alto riesgo de trabajar en condiciones psicosociales laborales adversas, y que estas condiciones se relacionan con indicadores negativos de salud. Entre el $54 \%$ y el $22 \%$ de la muestra (según los dos criterios de evaluación de los factores psicosociales laborales) presentan condiciones psicosociales laborales negativas, lo cual es una prevalencia comparable a la encontrada en otros estudios (Schnall et al., 2000). Sobre los factores psicosociales reportados vale la pena destacar que los maestros colombianos contrastan con otros grupos nacionales e internacionales, por percibir más demandas pero también más control de las mismas, más esfuerzo y sobrecompromiso. A pesar de esto, alrededor de un cuarto de los participantes percibe un desbalance entre esas demandas y sus posibilidades de control, y entre el esfuerzo que hacen y las recompensas que reciben a cambio. Además, parecen comprometerse demasiado con sus trabajos, lo cual ha demostrado incrementar la percepción de esfuerzo y los riesgos para la salud.

Según las correlaciones calculadas, el exceso de demandas, el desbalance demanda-control pero especialmente el esfuerzo, la falta de recompensas y el estilo demasiado comprometido de afrontar el trabajo son los factores que más se relacionan con un bajo nivel de salud en los docentes. En particular, los indicadores de estrés laboral (mayor percepción de desbalance entre demanda y control y de desbalance esfuerzo-recompensa) se relacionaron significativamente con experiencia de ansiedad y psicosomatización, y en menor medida con depresión y disfunción social. A pesar de que este estudio no permite hacer afirmaciones de causalidad, hay suficientes evidencias previas para pensar que las condiciones psicosociales laborales están generando este malestar en los docentes. Nos interesa destacar que entre los dos modelos logran explicar el 35\% de la varianza en la salud mental, lo cual no es un porcentaje despreciable teniendo en cuenta los múltiples factores que tienen la capacidad de afectar esta variable.

Los resultados de este estudio coinciden con los reportados por Diazgranados et al. (2006) en una muestra de docentes, aunque el grupo aquí estudiado laboraba en el sector privado y el evaluado en el 2006 en el sector público. Los resultados de este estudio también coinciden con la mayor parte de los estudios publicados acerca del estrés laboral de docentes escolares que señala que su mayor impacto es en la salud mental.

Los resultados aquí reportados no mostraron, sin embargo, en el grupo total, una relación directa entre los factores psicosociales laborales (medidos con base en dos modelos distintos y complementarios) y la tensión arterial. Los pocos estudios previos en los que no se halló ninguna relación entre el estrés laboral y la tensión arterial fueron en su mayoría realizados con grupos ocupaciona- 
les específicos, únicos (p. ej., Ragland, Winkleby, Schwalbe, Holman, Morse, Syme \& Fisher, 1987), en vez de grupos compuestos de personas con diversas ocupaciones que difieren de manera amplia en las condiciones psicosociales que caracterizan sus labores. Algunos autores afirman que cuando se evalúa una sola ocupación, el nivel de tensión laboral reportado por las diversas personas resulta ser muy homogéneo lo cual no permitiría identificar fácilmente correlaciones con la tensión arterial. Según Landsbergis et al. (2008), los pocos estudios previos que no han logrado demostrar una relación entre la tensión laboral y la presión sanguínea han compartido esta característica.

Otras posibles explicaciones para la falta de relación entre el estrés laboral y la tensión arterial, inesperada en este grupo de maestros escolares, podrían ser:

1. La muestra evaluada parece ser en general grupo sano (p. ej., índice de masa corporal y tensión arterial promedio) lo cual hace más difícil encontrar una relación clara entre cualquier tipo de condición vital negativa y un problema de salud física como la tensión arterial.

2. El tipo de condiciones laborales que experimentan los docentes puede hacerlos más vulnerables a impactos de tipo psicológico pero no físico. Algunas ocupaciones han mostrado ser más sensibles al riesgo cardiovascular (choferes, enfermeras) y podría ser que los maestros no pertenecen a este grupo. Varios estudios han demostrado que uno de los aspectos que caracteriza las ocupaciones con alto riesgo de tensión arterial alta como consecuencia del estrés laboral, es el tener que estar con mucha frecuencia vigilante para evitar las consecuencia fatales o desastrosas de un error en el trabajo (Schnall et al., 2000). Las descripciones que los mismos docentes hacen de sus labores señalan muchos aspectos estresantes, pero este no parece ser uno de ellos.

3. Algunas variables mediadoras o moderadoras podrían explicar el resultado negativo. Es posible que para diferenciar los maestros con estrés laboral que reaccionan con elevaciones de la presión arterial de aquellos que no lo hacen, se requieran otros criterios, además de las condiciones psicosociales laborales. Por ejemplo, la presión arterial de maestros que experimentan tensión laboral, pero además se caracterizan por presentar una mayor reactividad cardiovascular en situaciones demandantes (menos controlables), es más alta según varios estudios previos (Steptoe \& Cropley, 2000; Steptoe, Cropley \& Joekes, 1999). Estos investigadores han encontrado en muestras de profesores, que los grupos de sujetos con tensión laboral alta y baja no difieren respecto a factores demográficos, índice de masa corporal, o actividad cardiovascular durante el reposo. Pero ante tareas incontrolables la presión arterial en el grupo con mayores demandas o mayor tensión laboral se incrementa más intensamente; las respuestas ante las tareas controlables no diferencian significativamente entre los grupos. Adicionalmente, tanto la tensión arterial sistólica como la diastólica no se diferencian entre los grupos durante un día laboral, pero disminuyen por la noche, más intensamente, en los sujetos con baja tensión laboral. Según estos autores, la tensión laboral se asocia con una más intensa respuesta de la tensión arterial durante tareas incontrolables pero no durante las controlables. Steptoe y Cropley (2000) y Steptoe, Cropley y Joekes (1999) concluyen que la reactividad cardiovascular durante situaciones estresantes y el estrés psicosocial sostenido pueden actuar mancomunadamente para incrementar el riesgo cardiovascular en individuos susceptibles. Según ellos, el fracaso de los sujetos con alta tensión laboral en mostrar una disminución de la presión arterial en las noches puede ser una manifestación de una carga alostática crónica.

4. Otras variables biomédicas, sociales o psicológicas, no medidas en este estudio, podrían actuar como moderadoras de la relación entre el estrés laboral y la tensión arterial. Nosotros medimos la necesidad de control (Román, 2003), y el conflicto mutuo entre la familia y el trabajo. Los resultados de estos análisis se reportan en otros artículos. 
5. El hecho de que la mayor parte de los participantes hayan sido mujeres, y que su presión arterial fuera significativamente más baja que la de los hombres (lo cual confirma datos en el sentido de que prevalencia de hipertensión arterial en trabajadores colombianos se sitúa entre el $16 \%$ y $26 \%$ para los hombres y entre el $9 \%$ y $12 \%$ para las mujeres) podría reducir la posibilidad de identificar cómo el estrés laboral afecta la tensión arterial además de indicar la necesidad de realizar análisis separados para cada sexo. Efectivamente, los análisis realizados de manera separada entre hombres y mujeres, confirmaron que sólo en los hombres se presentó una relación negativa entre la presión arterial sistólica y las recompensas recibidas a cambio del esfuerzo realizado en el trabajo. El hecho de que tener menores recompensas afecte especialmente a los hombres, y no a las mujeres, podría interpretarse como una consecuencia de que muchos de ellos, en general, se consideren aún los principales proveedores de la familia. Las recompensas en el modelo de Siegrist se entienden como un pago justo, estabilidad laboral, posibilidades de ascenso, entre otras, aspectos todos, que de ser negativos, pueden afectar esta responsabilidad que socialmente sigue siendo predominantemente masculina. Si las condiciones de trabajo amenazan la posibilidad de cumplir con esta función, es posible pensar que estas afecten más a quienes se considera más responsables de ella. En el caso de las mujeres, además de la variable demográfica edad y del factor de riesgo biomédico IMC, sólo el factor psicosocial "uso de habilidades" logró explicar una proporción pequeña de la varianza de la tensión arterial sistólica. En el caso de las docentes, percibir que en su trabajo aprenden, son creativas, que su trabajo requiere buen nivel de habilidad, entre otros, son factores que se asocian con niveles de tensión arterial menores. Estos últimos hallazgos coinciden con lo señalado en diversos estudios previos, en Colombia y fuera de ella, que señalan que hombres y mujeres pueden ser afectados de manera distinta por los diversos factores psicosociales que caracterizan su ambiente laboral (Calnan et al., 2000; Kouvonen et al., 2005; Marulanda, 2007; Peter et al., 2002).

Finalmente, es importante señalar que hay algunas características de la muestra estudiada o de las condiciones en que se realizó este estudio que limitan las conclusiones obtenidas y la generalización de las mismas. Por ejemplo, este estudio fue transversal y no longitudinal, lo cual no permite hacer afirmaciones de causalidad. El tamaño de la muestra y el hecho de que ésta labore sólo en el sector privado de la educación en Bogotá, limitan las conclusiones que pueden obtenerse de estos datos. Las edades de los alumnos con que se trabaja fue una variable que no se midió en este estudio, pero que sería interesante evaluar como factor que puede afectar el nivel de estrés que se experimenta como docente.

Diversos estudios señalan que entre más mediciones de la presión arterial puedan hacerse al día, será más factible aproximarse a valores que representen las condiciones reales de las personas evaluadas. En este estudio se hicieron cuatro mediciones, siguiendo el protocolo sugerido por Schnall et al. (2000), sin embargo, eventualmente un mayor número de mediciones hubiera aumentado la precisión de nuestra estimación de la presión arterial de los maestros estudiados.

Futuros estudios deberán, además de aumentar el tamaño de la muestra y el número de mediciones de la presión arterial (o contemplar la posibilidad de monitorear ambulatoriamente la misma), evaluar el papel moderador de distinto tipo de variables, tanto psicológicas, como sociales y biológicas. Complementar los datos cuantitativos con entrevistas y observaciones podría también aumentar la exactitud de las afirmaciones que se hagan acerca del estrés laboral de maestros escolares y de su impacto en la salud de esta población.

\section{Referencias}

Arango, A. (2007). Análisis comparativo de propiedades psicométricas de los cuestionarios ERI y JCQ en una muestra de enfermeras y una de conductores de bus. 
Tesis de pregrado no publicada, Universidad de los Andes, Bogotá, Colombia.

Association for the Advancement of Medical Instrumentation. Disponible en http://www.healthcare. omron.co.jp/english/vali_us.php

Belkic, K. (2000). Occupational Stress Index: An introduction. Recuperado el 8 de octubre, 2008, de http:// www.workhealth.org

Calnan, M., Wainwrite, D. \& Almond, D. (2000). Job Strain, effort-reward imbalance and mental distress: A study of occupations in general medical practice. Work E Stress, 14(4), 297-311.

Cedillo, L. \& Karasek, R. (2003). Reliability and Validity of the Spanish Version of the Job Content Questionnaire Among Maquiladora Women Workers. JCQCenter. Disponible en www.jcqcenter.org

Cedillo, L. (2005). Estudio de los Factores Psicosociales laborales entre trabajadoras de la Industria Maquiladora de Exportación en Sonora México. En A. Juárez-García \& P. J. A. Ramírez (Eds.), Estrés Psicosocial en el Trabajo (EPT): Dónde y Quienes estamos en México. Compartiendo experiencias con investigadores de EPT en Estados Unidos (pp. 81 117). México: FES Iztacala.

Colombia, Defensoría del Pueblo (2008). Disponible en http://www.defensoria.org.co/red/anexos/pdf/11/ salud/i1_2008.pdf

Colombia, Diario Oficial, 47.059, miércoles 23 de julio de 2008.

Colombia, Ministerio de la Protección Social (2003). Estudio Nacional de Salud Mental.

Cropley, M. \& Steptoe, A. (1999, March). Job Strain, Blood Pressure and Responsivity to Uncontrollable Stress. Presented in the Work Stress \& Health: Organization of Work in a Global Economy, Baltimore, Maryland.

Diazgranados, S., González, C. \& Jaramillo, R. (2006). Aproximación a las problemáticas psicosociales y a los saberes y habilidades de los docentes del Distrito. Revista de Estudios Sociales, 23, 45-55.

European Society of Hypertension. Disponible en http:// www.eshonline.org

Fernández-Castro, J. \& Doval, E. (1994). Efectos del estrés docente sobre los hábitos de salud. Ansiedad y Estrés, 0, 127-133.
Fernández-López, J. A., Siegrist, J., Rôdel, A. \& Hernández-Mejía, R. (2003). El estrés laboral: un nuevo factor de riesgo. ¿Qué sabemos y qué podemos hacer? Atención Primaria, 31, 524-526.

Gómez, V. (2004). Estrés y Salud en mujeres que desempeñan múltiples roles (Número monográfico sobre psicología de la salud de la mujer). Avances en Psicología Latinoamericana, 22, 117-128.

Gómez, V. (2006). Quality of work and family roles and its relationship with health indicators in men and women. Sex Roles, 55 (11-12), 787-799.

Guerrero, E. (2003). Análisis pormenorizado de los grados de burnout y técnicas de afrontamiento del estrés docente en profesorado universitario. Anales de Psicología, 19(1), 145-158.

Hernández S., Baptista P. \& Collado, C. (1998). Metodología de la Investigación. México: McGraw Hill.

Hernández, D., Salazar, A. \& Gómez, V. (2004). Relación entre los aspectos psicosociales del ambiente de trabajo y el riesgo cardiovascular en hombres. Revista Latinoamericana de Psicología, 36(1), 107. 123.

Juárez-García, A. (2005). Factores psicosociales relacionados con la salud mental en profesionales de Servicios Humanos en México. Revista Chilena Ciencia \&O Trabajo, 14, 190-196.

Karasek, R. (1985). Job Content Questionnaire and user's guide. Lowell: University of Massachusetts Lowell, Department of Work Environment.

Karasek, R. (1998). Demand/control model: A social, emotional and physiological approach to stress risk and active behavior development. Encyclopedia of Occupational Health and Safety (p. 346). Sweden: Organización Internacional del Trabajo.

Karasek, R., Brisson, Ch., Kawakami, N., Houtman, I., Bongers, P. \& Amick, B. (1998). The Job Content Questionnaire (JCQ): An instrument for internationally comparative assessments of psychosocial job characteristics. Journal of Occupational Health Psychology, 3(4), 332-355.

Kouvonen, A., Kivimäki, M., Cox, S., Poikolainen, K. \& Cox, T. (2005). Job strain, effort-reward imbalance, and heavy drinking: A study in $40851 \mathrm{em}$ ployees. Journal of Occupational and Environmental Medicine, 47(5), 503-514. 
Landsbergis, P. A., Schnall. P. L., Belkić, K. L., Schwartz, J. E., Baker, D. \& Pickering, T. G. (2008). Work conditions and masked (hidden) hypertensioninsights into the global epidemic of hypertension. Scandinavian Journal of Work and Environmental Health, 6 (Suppl.), 41-51.

Marcías, M., Fernández, J., Hernández, R., Cueto, A., Rancaño, I. \& Siegrist, J. (2003). Evaluación del estrés laboral en trabajadores de un hospital público español. Estudio de las propiedades psicométricas de la versión española del modelo $<<$ Desequilibrio Esfuerzo-Recompensa $>>$. Medicina clínica, 120(17), 652-657.

Marulanda, I. C. (2007). Estrés Laboral. Enemigo silencioso de la salud mental y la satisfacción con la vida. Bogotá: Colección Prometeo, Universidad de los Andes, CESO, Departamento de Psicología.

Moreno Jiménez, B., Garrosa Hernández, E. \& González Gutiérrez, J. L. (2000). La evaluación del estrés y el burnout del profesorado: el CBP-R. Revista de Psicología del Trabajo y de las Organizaciones, 16(2), 151-171.

Oramas, A., Rodríguez, R., Almirall, P. J., Huerta, J. \& Vergara, A. (2003). Algunas Causas de Estrés Psicológico en Maestros del Municipio Arroyo Naranjo, Cuba. Revista Cubana de Salud y Trabajo, $4(1-2), 8-12$.

Pérez, L. A. \& Gómez, V. (2005, marzo). Psychosocial work related factors, stress and acute myocardial infarct in Colombian working people. Poster presentado en el Fourth Internacional Conference on Work Environment and cardiovascular Diseases. Newport Beach, CA, EE.UU.

Peter, R., Siegrist, J., Hallqvist, J., Reuterwall, C. \& Theorell, T. (2002). Psychosocial work environment and myocardial infarction: Improving risk estimation by combining two complementary job stress models in the SHEEP Study. Journal of Epidemiology and Community Health, 56, 294-300.

Posada-Villa, J., Aguilar-Gaxiola, S., Magaña, C. \& Gómez, L. C. (2004). Prevalencia de trastornos mentales y uso de servicios: resultados preliminares del Estudio Nacional de Salud Mental. Colombia, 2003. Revista Colombiana de Psiquiatría, 33(3), 241-62.
Ragland, D. R., Winkleby, M. A., Schwalbe, J., Holman, B. L., Morse, L., Syme, S. L. \& Fisher, J. M. (1987). Prevalence of hypertension in bus drivers. International Journal of Epidemiology, 16, 208-214.

Román, J., Romero, R. \& Ramírez, A. (2003). Necesidad de control sobre el trabajo como factor de riesgo de hipertensión arterial. Psicología y Salud, $13(1), 37-46$.

Schnall, P., Belkic, K., Landsbergis, P. \& Baker, D. (2000). The Workplace and Cardiovascular Disease. Occupational Medicine, 15(1), 1-6.

Schnall, P., \& Belkic, K. (2000). Point estimates of Blood Pressure at the Worksite. In P. Schnall, K. Belkic, P. Landsbergis \& D. Baker (Eds.), The Workplace and Cardiovascular Disease. Occupational Medicine, 15(1), 203-212.

Schaufeli, W. B. \& Enzmann, D. (1998). The burnout companion to study and practice: A critical analysis. London: Taylor \& Francis.

Siegrist, J. (2002). Effort-Reward Imbalance at work and health. En P. L. Perrewé \& D. C. Ganster (Eds.), Historical and Current Perspectives on Stress and Health (Vol. 2, pp. 261-291). Amsterdam: Elsevier Science.

Siegrist, J. \& Peter, R. (2000). The Effort-Reward Imbalance Model. Occupational Medicine, 1, 83-86.

Siegrist, J., Starke, D., Chandola, T., Godin, I., Marmot, M., Niedhammer, I. \& Peter, R. (2004). The measurement of Effort-Reward Imbalance at work: European comparisons. Social Science $\mathcal{E}$ Medicine, 58(8), 1483-1499.

Steptoe, A., \& Cropley, M. (2000). Persistent high job demands and reactivity to mental stress predict future ambulatory blood pressure. Journal of $\mathrm{Hy}$ pertension, 18(5), 581-586.

Steptoe, A., Cropley, M. \& Joekes, K. (1999). Job strain, blood pressure and response to uncontrollable stress. Journal of Hypertension, 17(2), 193-200.

Van Der Doef, M. \& Maes, S. (1999) The Job DemandControl (-Support) Model and psychological wellbeing: A review of 20 years of empirical research. Work and Stress, 13(2), 87-114. 